\title{
Topological and differentiable sphere theorems for complete submanifolds
}

\author{
Hong-Wei Xu AND EN-TAO ZHaO
}

\begin{abstract}
We investigate topological and differentiable structures of submanifolds under extrinsic restrictions. We first obtain a topological sphere theorem for compact submanifolds in a Riemannian manifold. Secondly, we prove an optimal differentiable sphere theorem for 4-dimensional complete submanifolds in a space form, which provides a partial solution of the smooth Poincaré conjecture. Finally, we prove some new differentiable sphere theorems for $n$-dimensional submanifolds in a Riemannian manifold.
\end{abstract}

\section{Introduction}

It seems very interesting to investigate curvature and topology of submanifolds in a Riemannian manifold. In contrast to the usual sphere theorems in Riemannian geometry, our results on topological and differentiable structures of submanifolds are obtained by imposing certain conditions on the second fundamental form.

Let $M$ be an $n$-dimensional complete manifold isometrically immersed in a Riemannian manifold $N^{n+p}$ with codimension $p$. Denote by $H$ and $S$ the mean curvature and squared length of the second fundamental form of $M$, respectively. Using nonexistence for stable currents on compact submanifolds of a sphere and the generalized Poincaré conjecture for dimension $n(\geq 5)$ proved by Smale [1], Lawson and Simons [2] obtained the following striking sphere theorem in 1970s.

Theorem A. Let $M^{n}$ be an $n$-dimensional oriented compact submanifold in the unit sphere $S^{n+p}$. Then

(i) if $n \neq 3,4$ and $S<2 \sqrt{n-1}$, then $M$ is homeomorphic to a sphere;

(ii) if $n=3,4$ and $S<n-1$, then $M$ is a homotopy sphere.

When $n \neq 3,4$, the following example shows that Lawson and Simons' pinching constant in Theorem A is optimal. 
Example 1.1. Set

$$
M=S^{n-1}\left(\frac{1}{\sqrt{1+\lambda^{2}}}\right) \times S^{1}\left(\frac{\lambda}{\sqrt{1+\lambda^{2}}}\right) \subset S^{n+1}
$$

where

$$
\lambda=\frac{n H+\sqrt{n^{2} H^{2}+4(n-1)}}{2(n-1)}, \quad H=\frac{\sqrt{n(n-1)^{1 / 2}-2(n-1)}}{n} .
$$

Then $M$ is an $n$-torus in $S^{n+1}\left(\subset S^{n+p}\right)$. Moreover, $M$ is a compact submanifold in $S^{n+p}$ with constant mean curvature $H$, whose squared length of the second fundamental form satisfies $S=2 \sqrt{n-1}$.

In 1997, Shiohama and $\mathrm{Xu}$ [4] proved the following topological sphere theorem for complete submanifolds in space forms by using the vanishing theorem for stable currents due to Lawson, Simons [2] and Xin [3].

Theorem B. Let $M^{n}$ be an $n$-dimensional oriented complete submanifold in a simply connected space form $F^{n+p}(c)$ with non-negative constant curvature c. Set

$$
\alpha(n, H, c)=n c+\frac{n^{3}}{2(n-1)} H^{2}-\frac{n(n-2)}{2(n-1)} \sqrt{n^{2} H^{4}+4(n-1) c H^{2}} .
$$

If $S$ satisfies $\sup _{M}(S-\alpha(n, H, c))<0$, then

(i) when $n \neq 3, M$ is homeomorphic to an $n$-dimensional sphere;

(ii) when $n=3, M$ is diffeomorphic to a 3-dimensional spherical space form.

From the following example, we see that the pinching condition in Theorem $\mathrm{B}$ is optimal.

Example 1.2. Let $M=S^{n-1}\left(1 /\left(1+\lambda^{2}\right)\right) \times S^{1}\left(\lambda /\left(\sqrt{1+\lambda^{2}}\right)\right) \subset S^{n+1}$ for $c=1$, and $M=S^{n-1}((n-1) / n H) \times \mathbf{R}^{1} \subset \mathbf{R}^{n+1}$ for $c=0$. Here $\lambda$ is as in Example 1.1 and $H$ is a non-negative constant for the case $c=1$, and $H$ is a positive constant for the case $c=0$. Then $M$ is an $n$-dimensional Clifford torus or generalized cylinder in $F^{n+1}(c)$. Moreover, $M$ is a complete submanifold in $F^{n+p}(c)$ with constant mean curvature $H$, whose squared length of the second fundamental form satisfies $S=\alpha(n, H, c)$. 
Notice that

$$
\inf _{H \geq 0} \alpha(n, H, 1)=2 \sqrt{n-1},
$$

we have the following consequence of Theorem B, which is an extension of Lawson-Simons' sphere theorem.

Theorem C. Let $M^{n}$ be an $n$-dimensional oriented complete submanifold in $S^{n+p}$. If $S$ satisfies $\sup _{M} S<2 \sqrt{n-1}$, then

(i) when $n \neq 3, M$ is homeomorphic to a sphere;

(ii) when $n=3, M$ is diffeomorphic to a spherical space form.

Let $U M$ denote the unit tangent bundle over $M$ and $U_{x} M$ its fiber over $x \in M$. In [5], Gauchman proved that if $M$ is a closed minimal submanifold and $\|h(u, u)\|^{2}<\frac{1}{3}$ for any $u \in U_{x} M$ at any point $x \in M$, where $h$ denotes the second fundamental form of $M$, then $M$ must be totally geodesic. The pinching constant $\frac{1}{3}$ is optimal in the sense that there are minimal submanifolds with $\|h(u, u)\|^{2}=\frac{1}{3}$, which are not totally geodesic. Following Leung's homotopy sphere theorem for compact oriented submanifolds in a sphere [6], $\mathrm{Xu}$ and Fang [7] got the following topological sphere theorem for complete submanifolds in a sphere.

Theorem D. Let $M^{n}$ be an $n$-dimensional oriented complete submanifold in the unit sphere $S^{n+p}$. If

$$
\|h(u, u)\|^{2}<\frac{1}{3}, \quad \forall u \in U M
$$

then $M$ is homeomorphic to an n-sphere. In particular, if $M$ is a submanifold with parallel mean curvature, then $M$ is congruent to the sphere $S^{n}\left(1 / \sqrt{1+H^{2}}\right)$.

The purpose of this paper is to prove some new pinching theorems for submanifolds in a Riemannian manifold. In particular, we obtain the following.

Theorem 1.1. Let $M^{n}$ be an $n(\geq 4)$-dimensional oriented complete submanifold in the unit sphere $S^{n+p}$. Then

(i) if $n=4,5,6$ and $\sup _{M} S<2 \sqrt{n-1}$, then $M$ is diffeomorphic to the standard unit $n$-sphere $S^{n}$; 
(ii) if $n \geq 7$ and $S<2 \sqrt{2}$, then $M$ is diffeomorphic to the standard unit $n$-sphere $S^{n}$.

When $n=4,5,6$, the pinching constant $2 \sqrt{n-1}$ in Theorem 1.1 is optimal.

Theorem 1.2. Let $M^{n}$ be an $n$-dimensional oriented complete submanifold in the unit sphere $S^{n+p}$. If

$$
\|h(u, u)\|^{2}<\frac{1}{3}, \quad \forall u \in U M
$$

then $M$ is diffeomorphic to $S^{n}$.

It should be emphasized that Theorems 1.1 and 1.2 are differentiable sphere theorems for complete submanifolds without assumption that $M$ is simply connected, which improve Theorems C and D.

Our paper is organized as follows. Some notations and several lemmas are prepared in Section 2. In Section 3, we obtain a topological sphere theorem for compact submanifolds in a Riemannian manifold. In Section 4, we prove an optimal differentiable sphere theorem for 4-dimensional complete submanifolds in a space form, which provides a partial solution of the smooth Poincaré conjecture. Finally, we prove some new differentiable pinching theorems for submanifolds in a Riemannian manifold, and complete the proof of Theorems 1.1 and 1.2.

\section{Some useful lemmas}

Let $M^{n}$ be an $n(\geq 4)$-dimensional Riemannian manifold. In 1988, using minimal surface techniques, Micallef and Moore [8] investigated relations between curvature and topology of a manifold, and proved the topological sphere theorem for point-wise 1/4-pinched manifolds. Further more, they proved the famous topological sphere theorem for compact and simply connected manifolds with positive isotropic curvature.

Recall that the Riemannian curvature operator at $p \in M$ is the selfadjoint linear endmorphism $\mathrm{Rm}: \wedge^{2} T_{p} M \longrightarrow \wedge^{2} T_{p} M$ defined by

$$
\langle\operatorname{Rm}(X \wedge Y),(Z \wedge W)\rangle=\langle R(X, Y) W, Z\rangle
$$

for $X, Y, Z, W \in T_{p} M$. Here $\langle$,$\rangle is the Riemannian metric, T_{p} M$ denotes the tangent space at $p$, and $R$ is the Riemannian curvature tensor of $M$. The Riemannian metric $\langle$,$\rangle can be extended either to a complex bilinear$ 
form ( , ) or a Hermitian inner product $\langle\langle\rangle$,$\rangle on T_{p} M \otimes C$. We extend the curvature operator to a complex linear map on $\wedge^{2} T_{p} M \otimes C$, also denoted by $\mathrm{Rm}$.

For each two-plane $\sigma \subseteq T_{p} M \otimes C$, we can define the complex sectional curvature $K(\sigma)$ by

$$
K(\sigma)=\frac{\langle\langle\operatorname{Rm}(X \wedge Y), X \wedge Y\rangle\rangle}{\|X \wedge Y\|^{2}},
$$

where $\{X, Y\}$ is a basis for $\sigma$. A two-plane $\sigma$ is called totally isotropic if $(Z, Z)=0$ for any $Z \in \sigma$. We say that $M$ has positive isotropic curvature if $K(\sigma)>0$ for all totally isotropic two-planes at any point in $M$. It was shown in [8] that $M$ has positive isotropic curvature if and only if for every orthonormal four-frame $\left\{e_{1}, e_{2}, e_{3}, e_{4}\right\}$ at any point in $M$ the inequality for curvature tensor of $M$

$$
R_{1313}+R_{1414}+R_{2323}+R_{2424}-2 R_{1234}>0
$$

holds, where $R_{i j k l}=\left\langle R\left(e_{i}, e_{j}\right) e_{l}, e_{k}\right\rangle,\left\{e_{1}, e_{2}, \ldots, e_{n}\right\}$ is a local orthonormal frame of $M$.

In [8], Micallef and Moore proved that any $n(\geq 4)$-dimensional compact and simply connected manifold with positive isotropic curvature must be homeomorphic to a sphere. Moreover, they observed that any $n(\geq 4)$ manifold with point-wise 1/4-pinched curvature must have positive isotropic curvature. In 1991, Chen [9] showed that a point-wise 1/4-pinched 4-manifold is diffeomorphic to a spherical space form. Recently, Brendle and Schoen $[10,11]$ proved a remarkable differentiable pinching theorem for point-wise 1/4-pinched Riemannian manifolds by developing the theory and techniques of Ricci flow [22-24]. More recently, Brendle [12] obtained the following useful result.

Lemma 2.1 [12]. Let $\left(M, g_{0}\right)$ be a compact Riemannian manifold of dimension $n \geq 4$. Assume that

$$
R_{1313}+\lambda^{2} R_{1414}+R_{2323}+\lambda^{2} R_{2424}-2 \lambda R_{1234}>0
$$

for all orthonormal four frames $\left\{e_{1}, e_{2}, e_{3}, e_{4}\right\}$ and all $\lambda \in[-1,1]$. Then the normalized Ricci flow with initial metric $g_{0}$

$$
\frac{\partial}{\partial t} g(t)=-2 R i c_{g(t)}+\frac{2}{n} r_{g(t)} g(t),
$$


exists for all time and converges to a constant curvature metric as $t \rightarrow \infty$. Here $r_{g(t)}$ denotes the mean value of the scalar curvature of $g(t)$.

Inequality (2.1) is closely related to the positivity of the isotropic curvature. In fact, $R_{1313}+\lambda^{2} R_{1414}+R_{2323}+\lambda^{2} R_{2424}-2 \lambda R_{1234}>0$ holds for all orthonormal four-frames $\left\{e_{1}, e_{2}, e_{3}, e_{4}\right\}$ and all $\lambda \in[-1,1]$ if and only if $M \times \mathbf{R}$ has positive isotropic curvature. It follows from Berger's inequality that every manifold with point-wise 1/4-pinched sectional curvatures satisfies the curvature condition (2.1) in Lemma 2.1. Hence, the differentiable 1/4-pinching theorem is a consequence of Lemma 2.1.

When $M$ is isometrically immersed into a Riemannian manifold $N^{n+p}$ with codimension $p \geq 1$, we choose a local orthonormal frame field $\left\{e_{1}, \ldots, e_{n+p}\right\}$ on $N^{n+p}$ such that, restricted to $M, e_{1}, \ldots, e_{n}$ are tangent to $M$. Let $\left\{\omega_{1}, \ldots, \omega_{n+p}\right\}$ be the dual frame field of $\left\{e_{1}, \ldots, e_{n+p}\right\}$. We shall make use of the following convention on the range of indices:

$1 \leq A, B, C, \cdots \leq n+p, \quad 1 \leq i, j, k, \cdots \leq n, \quad n+1 \leq \alpha, \beta, \gamma, \cdots \leq n+p$.

Then we have the Gauss equation

$$
R_{i j k l}=K_{i j k l}+\sum_{\alpha}\left(h_{i k}^{\alpha} h_{j l}^{\alpha}-h_{i l}^{\alpha} h_{j k}^{\alpha}\right)
$$

where $K_{A B C D}$ is the Riemannian curvature tensor of $N^{n+p}$ and $h=$ $\sum_{\alpha, i, j} h_{i j}^{\alpha} \omega_{i} \otimes \omega_{j} \otimes e_{\alpha}$ is the second fundamental form of $M$. Let $U M$ denote the unit tangent bundle on $M$ and $U_{x} M$ its fiber over $x \in M$. Then $U M=$ $\bigcup_{x \in M} U_{x} M$, where $U_{x} M=\left\{u \in T_{x} M ;\|u\|=1\right\}$.

Lemma 2.2. Let $M^{n}$ be a submanifold in a Riemannian manifold $N^{n+p}$. Then

$$
\|h(u, v)\| \leq h_{x}
$$

for all unit vectors $u, v \in U_{x} M$ at each point $x \in M$, where $h_{x}:=\max _{w \in U_{x} M}$ $\|h(w, w)\|$.

Proof. If $u \neq \pm v$, set $y=\frac{u+v}{\|u+v\|}$ and $z=\frac{u-v}{\|u-v\|}$. Then

$$
\begin{aligned}
h(u, v) & =\frac{1}{4}[h(u+v, u+v)-h(u-v, u-v)] \\
& =\frac{1}{4}\left[\|u+v\|^{2} h(y, y)-\|u-v\|^{2} h(z, z)\right] .
\end{aligned}
$$


Using the triangle inequality we obtain from (2.3)

$$
\begin{aligned}
\|h(u, v)\| & \leq \frac{1}{4} h_{x}\left(\|u+v\|^{2}+\|u-v\|^{2}\right) \\
& \leq h_{x}
\end{aligned}
$$

If $u= \pm v$, then $\|h(u, v)\|=\|h(u, u)\| \leq h_{x}$. This together with (2.4) completes the proof of the lemma.

The following algebraic lemma will be used in the proof of our sphere theorems.

Lemma 2.3. Let $T=\left(a_{i j}\right)_{4 \times 4}$ be a real symmetric matrix. Set $f(a)=a_{11} a_{33}-\left(a_{13}\right)^{2}+a_{11} a_{44}-\left(a_{14}\right)^{2}+a_{22} a_{33}-\left(a_{23}\right)^{2}+a_{22} a_{44}-\left(a_{24}\right)^{2}$ $-2 a_{13} a_{24}+2 a_{14} a_{23}$, where $a=\left(a_{11}, a_{12}, \cdots, a_{44}\right)$. Then

$$
\frac{1}{2}\left(\sum_{i=1}^{4} a_{i i}\right)^{2}-\sum_{i, j=1}^{4} a_{i j}^{2} \leq f(a) \leq \frac{1}{4}\left(\sum_{i=1}^{4} a_{i i}\right)^{2}
$$

Proof. Applying the Lagrange multiplier method, we compute the extreme value of $f(a)$ with constraints

$$
\sum_{i} a_{i i}=A
$$

and

$$
\sum_{i}\left(a_{i i}\right)^{2}+2 \sum_{i<j}\left(a_{i j}\right)^{2}=B
$$

where $B=\sum_{i, j} a_{i j}^{2}$. Consider the function

$$
F=f(a)+m_{1}\left(\sum_{i}\left(a_{i i}\right)^{2}+2 \sum_{i<j}\left(a_{i j}\right)^{2}-B\right)+m_{2}\left(\sum_{i} a_{i i}-A\right)
$$

where $m_{1}$ and $m_{2}$ are the Lagrange multipliers. Suppose that $f(a)$ attains the extreme value at point $\dot{a}=\left(\dot{a}_{11}, \dot{a}_{12}, \cdots, \dot{a}_{44}\right)$. Then at $\dot{a}$ we have

$$
\begin{aligned}
\frac{\partial F}{\partial a_{11}} & =\dot{a}_{33}+\dot{a}_{44}+2 m_{1} \dot{a}_{11}+m_{2}=0, \\
\frac{\partial F}{\partial a_{22}} & =\dot{a}_{33}+\dot{a}_{44}+2 m_{1} \dot{a}_{22}+m_{2}=0,
\end{aligned}
$$




$$
\begin{aligned}
\frac{\partial F}{\partial a_{33}} & =\dot{a}_{11}+\dot{a}_{22}+2 m_{1} \dot{a}_{33}+m_{2}=0, \\
\frac{\partial F}{\partial a_{44}} & =\dot{a}_{11}+\dot{a}_{22}+2 m_{1} \dot{a}_{44}+m_{2}=0, \\
\frac{\partial F}{\partial a_{12}} & =4 m_{1} \dot{a}_{12}=0 \\
\frac{\partial F}{\partial a_{34}} & =4 m_{1} \dot{a}_{34}=0, \\
\frac{\partial F}{\partial a_{13}} & =-2 \dot{a}_{13}-2 \dot{a}_{24}+4 m_{1} \dot{a}_{13}=0 \\
\frac{\partial F}{\partial a_{14}} & =-2 \dot{a}_{14}+2 \dot{a}_{23}+4 m_{1} \dot{a}_{14}=0 \\
\frac{\partial F}{\partial a_{23}} & =-2 \dot{a}_{23}+2 \dot{a}_{14}+4 m_{1} \dot{a}_{23}=0 \\
\frac{\partial F}{\partial a_{24}} & =-2 \dot{a}_{24}-2 \dot{a}_{13}+4 m_{1} \dot{a}_{24}=0 \\
\frac{\partial F}{\partial m_{1}} & =\sum_{i}\left(\dot{a}_{i i}\right)^{2}+2 \sum_{i<j}\left(\dot{a}_{i j}\right)^{2}-B=0 \\
\frac{\partial F}{\partial m_{2}} & =\sum_{i} \dot{a}_{i i}-A=0 .
\end{aligned}
$$

When $m_{1}=0$, from equalities (2.7) we obtain

$$
\dot{a}_{11}+\dot{a}_{22}=\dot{a}_{33}+\dot{a}_{44}, \dot{a}_{13}=-\dot{a}_{24}, \dot{a}_{14}=\dot{a}_{23} .
$$

Then

$$
f(\dot{a})=\left(\dot{a}_{11}+\dot{a}_{22}\right)\left(\dot{a}_{33}+\dot{a}_{44}\right) .
$$

From (2.7) we obtain

$$
\dot{a}_{11}+\dot{a}_{22}=\dot{a}_{33}+\dot{a}_{44}=\frac{A}{2} .
$$

Hence

$$
f(\dot{a})=\frac{A^{2}}{4} .
$$


When $m_{1} \neq 0$, it follows from $(2.7)$ that

$$
\dot{a}_{11}=\dot{a}_{22}, \quad \dot{a}_{33}=\dot{a}_{44}, \quad \dot{a}_{12}=0, \quad \dot{a}_{34}=0, \quad \dot{a}_{13}=\dot{a}_{24}, \quad \dot{a}_{14}=-\dot{a}_{23} .
$$

Combining equalities above we have

$$
\begin{aligned}
f(\dot{a}) & =4 \dot{a}_{11} \dot{a}_{33}-4\left(\dot{a}_{13}\right)^{2}-4\left(\dot{a}_{14}\right)^{2}, \\
\sum_{i<j}\left(\dot{a}_{i j}\right)^{2} & =2\left(\dot{a}_{13}\right)^{2}+2\left(\dot{a}_{14}\right)^{2} .
\end{aligned}
$$

Since $B=\sum_{i}\left(\dot{a}_{i i}\right)^{2}+2 \sum_{i<j}\left(\dot{a}_{i j}\right)^{2}$, it follows that

$$
f(\dot{a})=2\left(\dot{a}_{11}+\dot{a}_{33}\right)^{2}-B .
$$

By (2.7) we have

$$
\dot{a}_{11}+\dot{a}_{33}=\frac{A}{2}
$$

Hence

$$
f(\dot{a})=\frac{A^{2}}{2}-B
$$

Denote by $f_{\max }$ and $f_{\min }$ the maximal and minimum values of function $f(a)$ with constraints (2.5) and (2.6), respectively. It is easy to see that $B \geq \frac{A^{2}}{4}$. This together with (2.8) and (2.9) implies that

$$
\begin{aligned}
& f_{\max }=\max \left\{\frac{A^{2}}{4}, \frac{A^{2}}{2}-B\right\}=\frac{A^{2}}{4}, \\
& f_{\text {min }}=\min \left\{\frac{A^{2}}{4}, \frac{A^{2}}{2}-B\right\}=\frac{A^{2}}{2}-B .
\end{aligned}
$$

This proves the lemma.

\section{A topological sphere theorem for compact submanifolds}

By using the vanishing theorem for stable currents on compact submanifolds of a sphere, Lawson and Simons [2] obtained a topological sphere theorem for submanifolds in the sphere. Later Shiohama and $\mathrm{Xu}$ [4] improved Lawson-Simons' sphere theorem. Recently, Fu and Xu [13] proved a vanishing theorem for homology groups of submanifolds in the hyperbolic space 
$H^{n+p}$. They also obtained a topological sphere theorem for submanifolds in $H^{n+p}$ by using their vanishing theorem. However, if the ambient space is a general Riemannian manifold, their method does not work. Now we prove a topological sphere theorem for submanifolds in a Riemannian manifold by using a different argument.

Theorem 3.1. Let $M^{n}$ be an $n(\geq 4)$-dimensional oriented compact submanifold in an $(n+p)$-dimensional Riemannian manifold $N^{n+p}$. Denote by $K(x, \pi)$ the sectional curvature of $N$ for tangent 2-plane $\pi \subset T_{x} N$ at point $x \in N$. Set $K_{\max }(x):=\max _{\pi \subset T_{x} N} K(x, \pi), K_{\min }(x):=\min _{\pi \subset T_{x} N} K(x, \pi)$. If

$$
S(x)<\frac{16}{3}\left(K_{\min }(x)-\frac{1}{4} K_{\max }(x)\right)+\frac{n^{2} H^{2}(x)}{n-2},
$$

for any $x \in M$, then $M$ has positive isotropic curvature and $\pi_{k}(M)=0$ for $2 \leq k \leq[n / 2]$. In particular, if $M$ is simply connected, then $M$ is homeomorphic to a sphere.

Proof. Suppose $\left\{e_{1}, e_{2}, e_{3}, e_{4}\right\}$ is an orthonormal four-frame. Then we have

$$
\begin{aligned}
R_{1313} & +R_{1414}+R_{2323}+R_{2424}-2 R_{1234} \\
= & K_{1313}+K_{1414}+K_{2323}+K_{2424}-2 K_{1234} \\
& +\sum_{\alpha}\left[h_{11}^{\alpha} h_{33}^{\alpha}-\left(h_{13}^{\alpha}\right)^{2}+h_{11}^{\alpha} h_{44}^{\alpha}-\left(h_{14}^{\alpha}\right)^{2}\right. \\
& \left.+h_{22}^{\alpha} h_{33}^{\alpha}-\left(h_{23}^{\alpha}\right)^{2}+h_{22}^{\alpha} h_{44}^{\alpha}-\left(h_{24}^{\alpha}\right)^{2}-2 h_{13}^{\alpha} h_{24}^{\alpha}+2 h_{14}^{\alpha} h_{23}^{\alpha}\right] .
\end{aligned}
$$

It follows from Lemma 2.3 that

$$
\begin{aligned}
\sum_{\alpha} & {\left[h_{11}^{\alpha} h_{33}^{\alpha}-\left(h_{13}^{\alpha}\right)^{2}+h_{11}^{\alpha} h_{44}^{\alpha}-\left(h_{14}^{\alpha}\right)^{2}\right.} \\
& \left.+h_{22}^{\alpha} h_{33}^{\alpha}-\left(h_{23}^{\alpha}\right)^{2}+h_{22}^{\alpha} h_{44}^{\alpha}-\left(h_{24}^{\alpha}\right)^{2}-2 h_{13}^{\alpha} h_{24}^{\alpha}+2 h_{14}^{\alpha} h_{23}^{\alpha}\right] \\
\geq & \sum_{\alpha}\left[\frac{\left(\sum_{i=1}^{4} h_{i i}^{\alpha}\right)^{2}}{2}-\sum_{i, j=1}^{4}\left(h_{i j}^{\alpha}\right)^{2}\right] .
\end{aligned}
$$


When $n=4$, from (3.2) we obtain

$$
\begin{aligned}
\sum_{\alpha} & {\left[h_{11}^{\alpha} h_{33}^{\alpha}-\left(h_{13}^{\alpha}\right)^{2}+h_{11}^{\alpha} h_{44}^{\alpha}-\left(h_{14}^{\alpha}\right)^{2}\right.} \\
& \left.+h_{22}^{\alpha} h_{33}^{\alpha}-\left(h_{23}^{\alpha}\right)^{2}+h_{22}^{\alpha} h_{44}^{\alpha}-\left(h_{24}^{\alpha}\right)^{2}-2 h_{13}^{\alpha} h_{24}^{\alpha}+2 h_{14}^{\alpha} h_{23}^{\alpha}\right] \\
& \geq 8 H^{2}-S .
\end{aligned}
$$

When $n \geq 5$, we extend $\left\{e_{1}, e_{2}, e_{3}, e_{4}\right\}$ to be an orthonormal frame $\left\{e_{1}, e_{2}, \cdots, e_{n}\right\}$. Set $S_{\alpha}=\sum_{i, j=1}^{n}\left(h_{i j}^{\alpha}\right)^{2}$. Then from (3.2) we obtain

$$
\begin{aligned}
& \sum_{\alpha}\left[h_{11}^{\alpha} h_{33}^{\alpha}-\left(h_{13}^{\alpha}\right)^{2}+h_{11}^{\alpha} h_{44}^{\alpha}-\left(h_{14}^{\alpha}\right)^{2}\right. \\
& \left.\quad+h_{22}^{\alpha} h_{33}^{\alpha}-\left(h_{23}^{\alpha}\right)^{2}+h_{22}^{\alpha} h_{44}^{\alpha}-\left(h_{24}^{\alpha}\right)^{2}-2 h_{13}^{\alpha} h_{24}^{\alpha}+2 h_{14}^{\alpha} h_{23}^{\alpha}\right] \\
& \geq \sum_{\alpha}\left[\frac{\left(\sum_{i=1}^{4} h_{i i}^{\alpha}\right)^{2}}{2}-\sum_{i, j=1}^{4}\left(h_{i j}^{\alpha}\right)^{2}\right] \\
& \geq \sum_{\alpha}\left[\frac{\left(\sum_{i=1}^{4} h_{i i}^{\alpha}\right)^{2}}{2}+\sum_{i, j=5}^{n}\left(h_{i j}^{\alpha}\right)^{2}-S_{\alpha}\right] \\
& \geq \sum_{\alpha}\left[\frac{\left(\sum_{i=1}^{4} h_{i i}^{\alpha}\right)^{2}}{2}+\frac{\left(\sum_{i=5}^{n} h_{i i}^{\alpha}\right)^{2}}{n-4}-S_{\alpha}\right] \\
& \geq \sum_{\alpha}\left[\frac{\left(\sum_{i=1}^{n} h_{i i}^{\alpha}\right)^{2}}{n-2}-S_{\alpha}\right] \\
& \quad=\frac{n^{2}}{n-2} H^{2}-S_{.}
\end{aligned}
$$

By Berger's inequality we have $K_{1234} \leq \frac{2}{3}\left(K_{\max }-K_{\min }\right)$. Combining (3.1), (3.3) and (3.4), we obtain

$$
\begin{aligned}
& R_{1313}+R_{1414}+R_{2323}+R_{2424}-2 R_{1234} \\
& \quad \geq 4 K_{\min }-\frac{4}{3}\left(K_{\max }-K_{\min }\right)+\frac{n^{2}}{n-2} H^{2}-S \\
& \quad \geq \frac{16}{3}\left(K_{\min }-\frac{1}{4} K_{\max }\right)+\frac{n^{2}}{n-2} H^{2}-S \\
& \quad>0
\end{aligned}
$$


From (3.5) we know that $M$ has positive isotropic curvature. By a theorem due to Micallef and Moore [8], we have $\pi_{k}(M)=0$ for $2 \leq k \leq[n / 2]$. In particular, if $M$ is simply connected, then $M$ is homeomorphic to a sphere. This completes the proof of Theorem 3.1.

If the Ricci curvature of $M$ is positive, then the universal cover of $M$ is compact. Hence we have following.

Corollary 3.1. Under the same assumption as in Theorem 3.1, if the Ricci curvature of $M$ is positive and

$$
S(x)<\frac{16}{3}\left(K_{\min }(x)-\frac{1}{4} K_{\max }(x)\right)+\frac{n^{2} H^{2}(x)}{n-2},
$$

for any $x \in M$, then the universal cover of $M$ is homeomorphic to a sphere.

Moreover, from Theorem 3.1 we have

Corollary 3.2. Let $M^{n}$ be an $n(\geq 4)$-dimensional oriented compact submanifold in an $(n+p)$-dimensional pinched Riemannian manifold whose sectional curvature satisfies $b \leq K_{M} \leq c$. If $S<\frac{16}{3}\left(b-\frac{1}{4} c\right)+\frac{n^{2} H^{2}}{n-2}$, then $M$ has positive isotropic curvature and $\pi_{k}(M)=0$ for $2 \leq k \leq[n / 2]$. In particular, if $M$ is simply connected, then $M$ is homeomorphic to a sphere.

Corollary 3.3. Let $M^{n}$ be an $n(\geq 4)$-dimensional oriented compact submanifold in an $(n+p)$-dimensional point-wise $\delta(>1 / 4)$-pinched Riemannian manifold $N^{n+p}$. Set $K_{\max }(x):=\max _{\pi \subset T_{x} N} K(x, \pi)$, where $K(x, \pi)$ is the sectional curvature of $N$ for tangent 2-plane $\pi\left(\subset T_{x} N\right)$ at point $x \in N$. If $S(x)<\frac{16}{3} K_{\max }(x)\left(\delta-\frac{1}{4}\right)+\frac{n^{2} H^{2}(x)}{n-2}$, for any $x \in M$, then $M$ has positive isotropic curvature and $\pi_{k}(M)=0$ for $2 \leq k \leq[n / 2]$. In particular, if $M$ is simply connected, then $M$ is homeomorphic to a sphere.

Corollary 3.4. Let $M^{n}$ be an $n(\geq 4)$-dimensional oriented compact submanifold in an $(n+p)$-dimensional space form $F^{n+p}(c)$ of constant curvature c. If $S<4 c+\frac{n^{2} H^{2}}{n-2}$, then $M$ has positive isotropic curvature and $\pi_{k}(M)=0$ for $2 \leq k \leq[n / 2]$. In particular, if $M$ is simply connected, then $M$ is homeomorphic to a sphere.

\section{Differentiable sphere theorems for complete submanifolds}

It is not currently known how many differentiable structures there are on the 4-sphere, beyond that there is at least one. There may be one, a finite 
number, or an infinite number. The claim that there is just one is known as the smooth Poincaré conjecture. Most mathematicians believe that this conjecture is false, i.e., there are more than one differentiable structure on the 4-sphere. In this section, we first prove following theorem.

Theorem 4.1. Let $M^{4}$ be a 4-dimensional oriented, simply connected and compact submanifold in the space form $F^{4+p}(c)$ with constant curvature $c$. If $S<4 c+8 H^{2}$, then $M$ is diffeomorphic to the standard unit 4-sphere $S^{4}$.

Proof. From Corollary 3.4, we know that $M$ has positive isotropic curvature. A theorem due to Hamilton [14] says that a 4-dimensional compact simply connected manifold with positive isotropic curvature is diffeomorphic to $S^{4}$. This proves Theorem 4.1 .

The following example shows that Theorem 4.1 is optimal in the case where $c>0$.

Example 4.1. Without loss of generality, we only consider the case $c=1$. Set $M=S^{2}\left(1 / \sqrt{1+\mu^{2}}\right) \times S^{2}\left(\mu / \sqrt{1+\mu^{2}}\right) \subset S^{5}$, where $\mu=H+\sqrt{H^{2}+1}$ and $H$ is a non-negative constant. Then $M$ is a simply connected Clifford torus in $S^{5}\left(\subset S^{4+p}\right)$. Moreover, $M$ is a compact submanifold in $S^{4+p}$ with constant mean curvature $H$, whose squared length of the second fundamental form satisfies $S=4+8 H^{2}$.

Remark. In fact, it follows from Theorem 3.1 that if $M^{4} \subset N^{4+p}$ is a 4 dimensional compact and simply connected submanifold satisfying $S(x)<$ $\frac{16}{3}\left(K_{\min }(x)-\frac{1}{4} K_{\max }(x)\right)+8 H^{2}(x)$ for any $x \in M$, then $M$ is diffeomorphic to $S^{4}$.

Now, we prove an optimal differentiable sphere theorem for 4-dimensional complete submanifolds in space forms, which provides a partial solution of the smooth Poincaré conjecture.

Theorem 4.2. Let $M^{4}$ be a 4-dimensional oriented complete submanifold in a simply connected space form $F^{4+p}(c)$ with nonnegative constant curvature c. If $\sup _{M}(S-\alpha(4, H, c))<0$, then $M$ is diffeomorphic to the standard unit 4-sphere $S^{4}$. 
Proof. Since $c \geq 0$, we have

$$
\begin{aligned}
& \alpha(n, H, c)=n c+\frac{n^{3}}{2(n-1)} H^{2}-\frac{n(n-2)}{2(n-1)} \sqrt{n^{2} H^{4}+4(n-1) c H^{2}} \\
& \leq n c+\frac{n^{3}}{2(n-1)} H^{2}-\frac{n(n-2)}{2(n-1)} \sqrt{n^{2} H^{4}}=n c+\frac{n^{2}}{n-1} H^{2} .
\end{aligned}
$$

This implies

$$
S<\alpha(4, H, c) \leq 4 c+8 H^{2} .
$$

By Theorem B, $M$ is a topological sphere. This together with Theorem 4.1 implies that $M$ is diffeomorphic to $S^{4}$.

It is seen from Example 1.2 that the pinching condition $\sup _{M}(S-\alpha$ $(4, H, c))<0$ in Theorem 4.2 is optimal. It seems very difficult to generalize Theorem 4.2 to higher dimensional cases.

During the past three decades, there are several sphere theorems for submanifolds (see $[2,4,6,13,15-21]$, etc.). However, all these results are either topological sphere theorems for submanifolds, or differentiable sphere theorems for hypersurfaces. Using Brendle's convergence result for the normalized Ricci flow, we prove following new differentiable pinching theorems for submanifolds in higher dimensions and codimensions.

Theorem 4.3. Let $M^{n}$ be an $n(\geq 4)$-dimensional oriented compact submanifold in an $(n+p)$-dimensional point-wise $\delta(>1 / 4)$-pinched Riemannian manifold $N^{n+p}$. Set $K_{\max }(x):=\max _{\pi \subset T_{x} N} K(x, \pi)$, where $K(x, \pi)$ is the sectional curvature of $N$ for tangent 2-plane $\pi\left(\subset T_{x} N\right)$ at point $x \in N$. If $S(x)<\frac{8 \sqrt{2}}{3} K_{\max }(x)\left(\delta-\frac{1}{4}\right)$, for any $x \in M$, then $M$ is diffeomorphic to a space form. In particular, if $M$ is simply connected, then $M$ is diffeomorphic to the standard unit $n$-sphere $S^{n}$.

Proof. It is sufficient to show that inequality (2.1) in Lemma 2.1 holds for all $\lambda \in \mathbf{R}$.

Suppose $\left\{e_{1}, e_{2}, e_{3}, e_{4}\right\}$ is an orthonormal four-frame and $\lambda \in \mathbf{R}$. By the Gauss equation, we have

$$
\begin{aligned}
& R_{1313}+\lambda^{2} R_{1414}+R_{2323}+\lambda^{2} R_{2424}-2 \lambda R_{1234} \\
& =K_{1313}+\lambda^{2} K_{1414}+K_{2323}+\lambda^{2} K_{2424}-2 \lambda K_{1234} \\
& \quad+\sum_{\alpha}\left[h_{11}^{\alpha} h_{33}^{\alpha}-\left(h_{13}^{\alpha}\right)^{2}+h_{22}^{\alpha} h_{33}^{\alpha}-\left(h_{23}^{\alpha}\right)^{2}\right]
\end{aligned}
$$




$$
\begin{aligned}
& +\lambda^{2} \sum_{\alpha}\left[h_{11}^{\alpha} h_{44}^{\alpha}-\left(h_{14}^{\alpha}\right)^{2}+h_{22}^{\alpha} h_{44}^{\alpha}-\left(h_{24}^{\alpha}\right)^{2}\right] \\
& -2 \lambda \sum_{\alpha}\left(h_{13}^{\alpha} h_{24}^{\alpha}-h_{14}^{\alpha} h_{23}^{\alpha}\right) .
\end{aligned}
$$

By Berger's inequality and Young's inequality, we have from (4.1)

$$
\begin{aligned}
& R_{1313}+\lambda^{2} R_{1414}+R_{2323}+\lambda^{2} R_{2424}-2 \lambda R_{1234} \\
\geq & \left(2+2 \lambda^{2}\right) \delta K_{\max }-\frac{4}{3}|\lambda|(1-\delta) K_{\max } \\
& -\sum_{\alpha}\left[\frac{\sqrt{2}}{2}\left(h_{11}^{\alpha}\right)^{2}+\frac{\sqrt{2}}{4}\left(h_{33}^{\alpha}\right)^{2}+\left(h_{13}^{\alpha}\right)^{2}+\frac{\sqrt{2}}{2}\left(h_{22}^{\alpha}\right)^{2}\right. \\
& \left.+\frac{\sqrt{2}}{4}\left(h_{33}^{\alpha}\right)^{2}+\left(h_{23}^{\alpha}\right)^{2}\right] \\
& -\lambda^{2}\left\{\sum _ { \alpha } \left[\frac{\sqrt{2}}{2}\left(h_{11}^{\alpha}\right)^{2}+\frac{\sqrt{2}}{4}\left(h_{44}^{\alpha}\right)^{2}+\left(h_{14}^{\alpha}\right)^{2}+\frac{\sqrt{2}}{2}\left(h_{22}^{\alpha}\right)^{2}\right.\right. \\
& \left.\left.+\frac{\sqrt{2}}{4}\left(h_{44}^{\alpha}\right)^{2}+\left(h_{24}^{\alpha}\right)^{2}\right]\right\} \\
& -\sum_{\alpha}\left(h_{24}^{\alpha}\right)^{2}-\lambda^{2} \sum_{\alpha}\left(h_{13}^{\alpha}\right)^{2}-\sum_{\alpha}\left(h_{14}^{\alpha}\right)^{2}-\lambda^{2} \sum_{\alpha}\left(h_{23}^{\alpha}\right)^{2} \\
& \left.\left.+\left(h_{24}^{\alpha}\right)^{2}+\left(h_{13}^{\alpha}\right)^{2}+\left(h_{23}^{\alpha}\right)^{2}\right]\right\} \\
& +\lambda^{2}\left\{\sum_{\alpha}+\frac{\sqrt{2}}{2}\left(h_{11}^{\alpha}\right)^{2}+\frac{\sqrt{2}}{2}\left(h_{44}^{\alpha}\right)^{2}+\left(h_{14}^{\alpha}\right)^{2}+\frac{\sqrt{2}}{2}\left(h_{22}^{\alpha}\right)^{2}\right. \\
& +K_{\max }-\frac{4}{3}|\lambda|(1-\delta) K_{\max } \\
& +\frac{\sqrt{2}}{2}\left(h_{11}^{\alpha}\right)^{2}+\frac{\sqrt{2}}{2}\left(h_{33}^{\alpha}\right)^{2}+\left(h_{13}^{\alpha}\right)^{2}+\frac{\sqrt{2}}{2}\left(h_{22}^{\alpha}\right)^{2} \\
& \left.+\left(h_{14}^{\alpha}\right)^{2}\right]
\end{aligned}
$$




$$
\begin{aligned}
& \geq\left(2+2 \lambda^{2}\right) \delta K_{\max }-\frac{4}{3}|\lambda|(1-\delta) K_{\max }-\frac{\sqrt{2}}{2} S-\lambda^{2} \frac{\sqrt{2}}{2} S \\
& =\frac{\sqrt{2}}{2}\left(1+\lambda^{2}\right)\left[2 \sqrt{2} \delta K_{\max }-\frac{4 \sqrt{2}}{3} \frac{|\lambda|}{1+\lambda^{2}}(1-\delta) K_{\max }-S\right] \\
& \geq \frac{\sqrt{2}}{2}\left(1+\lambda^{2}\right)\left[\frac{8 \sqrt{2}}{3} K_{\max }\left(\delta-\frac{1}{4}\right)-S\right] \\
& >0 .
\end{aligned}
$$

It follows from Lemma 2.1 that $M$ is diffeomorphic to a space form. In particular, if $M$ is simply connected, then $M$ is diffeomorphic to $S^{n}$. This completes the proof of Theorem 4.3.

Consequently, we have the following.

Corollary 4.1. Let $M^{n}$ be an $n(\geq 4)$-dimensional oriented compact submanifold in an $(n+p)$-dimensional $\delta\left(>\frac{1}{4}\right)$-pinched Riemannian manifold $N^{n+p}$ whose sectional curvature satisfying $\delta \leq K_{N} \leq 1$. If $S<\frac{8 \sqrt{2}}{3}\left(\delta-\frac{1}{4}\right)$, then $M$ is diffeomorphic to a space form. In particular, if $M$ is simply connected, then $M$ is diffeomorphic to the standard unit n-sphere $S^{n}$.

Proof of Theorem 1.1. (i) When $n=4,5,6$, we have

$$
\inf _{H \geq 0} \alpha(n, H, 1)=2 \sqrt{n-1} .
$$

It's well known that there exists unique differentiable structure on $S^{n}$, $n=5,6$. This together with Theorem 4.2 and Theorem $\mathrm{C}$ implies that $M$ is diffeomorphic to a sphere.

(ii) When $n \geq 7$, it is seen from Theorem $\mathrm{C}$ that $M$ is a topological sphere. On the other hand, it follows from Corollary 4.1 that $M$ is diffeomorphic to a space form. Hence $M$ is diffeomorphic to $S^{n}$. This completes the proof of Theorem 1.1.

Motivated by Theorem D, we will prove a new differentiable pinching theorem for complete submanifolds in a point-wise $\delta$-pinched manifold. The following lemma will be used in the proof of the differentiable pinching theorem. 
Lemma 4.1. Let $M^{n}$ be an $n(\geq 4)$-dimensional submanifold in an $(n+p)$ dimensional point-wise $\delta(>1 / 4)$-pinched Riemannian manifold. If

$$
\|h(u, u)\|^{2}<\frac{4}{9} K_{\max }\left(\delta-\frac{1}{4}\right), \quad \forall u \in U_{x} M
$$

then inequality (1) is satisfied for all orthonormal four-frames $\left\{e_{1}, e_{2}, e_{3}, e_{4}\right\}$ and all $\lambda \in \mathbf{R}$.

Proof. For any point $x \in M$, let $h_{x}:=\max _{w \in U_{x} M}\|h(w, w)\|$. Suppose $\left\{e_{1}, e_{2}, e_{3}, e_{4}\right\}$ is an orthonormal four-frame and $\lambda \in \mathbf{R}$. By the Gauss equation, we have

$$
\begin{aligned}
R_{1313} & +\lambda^{2} R_{1414}+R_{2323}+\lambda^{2} R_{2424}-2 \lambda R_{1234} \\
= & K_{1313}+\left\langle h\left(e_{1}, e_{1}\right), h\left(e_{3}, e_{3}\right)\right\rangle-\left\|h\left(e_{1}, e_{3}\right)\right\|^{2} \\
& +\lambda^{2}\left[K_{1414}+\left\langle h\left(e_{1}, e_{1}\right), h\left(e_{4}, e_{4}\right)\right\rangle-\left\|h\left(e_{1}, e_{4}\right)\right\|^{2}\right] \\
& +\left[K_{2323}+\left\langle h\left(e_{2}, e_{2}\right), h\left(e_{3}, e_{3}\right)\right\rangle-\left\|h\left(e_{2}, e_{3}\right)\right\|^{2}\right] \\
& +\lambda^{2}\left[K_{2424}+\left\langle h\left(e_{2}, e_{2}\right), h\left(e_{4}, e_{4}\right)\right\rangle-\left\|h\left(e_{2}, e_{4}\right)\right\|^{2}\right] \\
& -2 \lambda\left[K_{1234}+\left\langle h\left(e_{1}, e_{3}\right), h\left(e_{2}, e_{4}\right)\right\rangle-\left\langle h\left(e_{1}, e_{4}\right), h\left(e_{2}, e_{3}\right)\right\rangle\right] .
\end{aligned}
$$

By (4.3) and Lemma 2.2, we have

$$
\begin{aligned}
& R_{1313}+\lambda^{2} R_{1414}+R_{2323}+\lambda^{2} R_{2424}-2 \lambda R_{1234} \\
& \geq K_{1313}-2 h_{x}^{2}+\lambda^{2}\left[K_{1414}-2 h_{x}^{2}\right] \\
& \quad+\left[K_{2323}-2 h_{x}^{2}\right]+\lambda^{2}\left[K_{2424}-2 h_{x}^{2}\right] \\
& \quad-2|\lambda|\left[\left|K_{1234}\right|+2 h_{x}^{2}\right] .
\end{aligned}
$$

This together with (4.2) and Berger's inequality implies

$$
\begin{aligned}
R_{1313} & +\lambda^{2} R_{1414}+R_{2323}+\lambda^{2} R_{2424}-2 \lambda R_{1234} \\
> & K_{\min }-\frac{8}{9} K_{\max }\left(\delta-\frac{1}{4}\right)+\lambda^{2}\left[K_{\min }-\frac{8}{9} K_{\max }\left(\delta-\frac{1}{4}\right)\right] \\
& +\left[K_{\min }-\frac{8}{9} K_{\max }\left(\delta-\frac{1}{4}\right)\right]+\lambda^{2}\left[K_{\min }-\frac{8}{9} K_{\max }\left(\delta-\frac{1}{4}\right)\right] \\
& -2|\lambda|\left[\frac{2}{3}(1-\delta) K_{\max }+\frac{8}{9} K_{\max }\left(\delta-\frac{1}{4}\right)\right]
\end{aligned}
$$




$$
\begin{aligned}
\geq & \delta K_{\max }-\frac{8}{9} K_{\max }\left(\delta-\frac{1}{4}\right)+\lambda^{2}\left[\delta K_{\max }-\frac{8}{9} K_{\max }\left(\delta-\frac{1}{4}\right)\right] \\
& +\left[\delta K_{\max }-\frac{8}{9} K_{\max }\left(\delta-\frac{1}{4}\right)\right]+\lambda^{2}\left[\delta K_{\max }-\frac{8}{9} K_{\max }\left(\delta-\frac{1}{4}\right)\right] \\
& -2|\lambda|\left[\frac{2}{3}(1-\delta) K_{\max }+\frac{8}{9} K_{\max }\left(\delta-\frac{1}{4}\right)\right] \\
= & \frac{1}{9}(\delta+2) K_{\max }\left(2+2 \lambda^{2}-4|\lambda|\right) \\
\geq & 0 .
\end{aligned}
$$

This completes the proof of the lemma.

Theorem 4.4. Let $M^{n}$ be an n-dimensional oriented complete submanifold in an $(n+p)$-dimensional point-wise $\delta(>1 / 4)$-pinched Riemannian manifold $N^{n+p}$. Set $K_{\max }(x):=\max _{\pi \subset T_{x} N} K(x, \pi)$, where $K(x, \pi)$ is the sectional curvature of $N$ for 2-plane $\pi \subset T_{x} N$ and point $x \in N$. If

$$
\|h(u, u)\|^{2}(x)<\frac{4}{9} K_{\max }(x)\left(\delta-\frac{1}{4}\right), \quad \forall u \in U_{x} M, x \in M,
$$

where $\inf _{x \in N} K_{\max }(x)>0$, then $M$ is diffeomorphic to a space form. In particular, if $M$ is simply connected, then $M$ is diffeomorphic to the standard unit $n$-sphere $S^{n}$.

Proof. Since $\|h(u, u)\|^{2}<\frac{4}{9}\left(\delta-\frac{1}{4}\right) K_{\max }$ for any $u \in U_{x} M$, we see from the Gauss equation that the sectional curvature of $M$ is bounded from below by $\frac{\delta+2}{9} \inf _{x \in M} K_{\max }(x) \geq \frac{\delta+2}{9} \inf _{x \in N} K_{\max }(x)>0$. By Myers' theorem, $M$ is a compact submanifold. When $n=2$, it is easy to see that $M$ is diffeomorphic to $S^{2}$ or $R P^{2}$. When $n=3$, the Hamilton theorem [22] says that $M$ is diffeomorphic to a spherical space form. When $n \geq 4$, by Lemma 2.1 and Lemma 4.1, we see that $M$ is diffeomorphic to a space form. In particular, if $M$ is simply connected, then $M$ must be diffeomorphic to the standard unit $n$-sphere $S^{n}$. This completes the proof of Theorem 4.4.

Consequently, we have

Corollary 4.2. Let $M^{n}$ be an $n$-dimensional oriented complete submanifold in an $(n+p)$-dimensional $\delta(>1 / 4)$-pinched Riemannian manifold. If

$$
\|h(u, u)\|^{2}<\frac{4}{9}\left(\delta-\frac{1}{4}\right), \quad \forall u \in U M
$$

then $M$ is diffeomorphic to a space form. In particular, if $M$ is simply connected, then $M$ is diffeomorphic to $S^{n}$. 
We are now in a position to prove Theorem 1.2 .

Proof of Theorem 1.2. It follows from Theorem D and Theorem 4.4 that $M$ is diffeomorphic to $S^{n}$. This completes the proof of Theorem 1.2.

Finally, we would like to propose the following conjecture.

Conjecture 4.1. Let $M^{n}$ be an $n(\geq 7)$-dimensional oriented complete submanifold in the $(n+p)$-dimensional unit sphere $S^{n+p}$. If $\sup _{M} S<2 \sqrt{n-1}$, then $M$ is diffeomorphic to $S^{n}$.

More general, we give the following.

Conjecture 4.2. Let $M^{n}$ be an $n(\geq 7)$-dimensional oriented complete submanifold in a simply connected space form $F^{n+p}(c)$ with non-negative constant curvature c. Set

$$
\alpha(n, H, c)=n c+\frac{n^{3}}{2(n-1)} H^{2}-\frac{n(n-2)}{2(n-1)} \sqrt{n^{2} H^{4}+4(n-1) c H^{2}} .
$$

If $\sup _{M}(S-\alpha(n, H, c))<0$, then $M$ is diffeomorphic to $S^{n}$.

Motivated by the topological sphere theorem due to Shiohama and $\mathrm{Xu}$ [19], we propose the following.

Conjecture 4.3. Let $M^{n}$ be an n-dimensional closed submanifold in a simply connected space form $F^{n+p}(c)$ with non-negative constant curvature $c$. Then there exists a positive constant $C_{n}$ depending only on $n$ such that if

$$
\int_{M}\left(S-n H^{2}\right)^{n / 2} \mathrm{~d} M<C_{n}
$$

then $M$ is diffeomorphic to $S^{n}$.

\section{Acknowledgments}

The authors would like to thank Professors Kefeng Liu and Feng Luo for their helpful discussions and valuable suggestions. Thanks also to the referee for his useful comments and suggestions. Research supported by the NSFC, Grant No. 10771187; the Trans-Century Training Programme Foundation for Talents by the Ministry of Education of China; and the Natural Science Foundation of Zhejiang Province, Grant No. 101037. 


\section{References}

[1] S. Smale, Generalized Poincaré's conjecture in dimensions greater than four, Ann. Math. (2), 74 (1961), 391-406.

[2] H. B. Lawson and J. Simons, On stable currents and their application to global problems in real and complex geometry, Ann. Math. 98 (1973), 427-450.

[3] Y. L. Xin, An application of integral currents to the vanishing theorems, Sci. Sinica Ser. A 27 (1984), 233-241.

[4] K. Shiohama and H. W. Xu, The topological sphere theorem for complete submanifolds, Compos. Math. 107 (1997), 221-232.

[5] H. Gauchman, Minimal submanifolds of a sphere with bounded second fundamental form, Trans. Am. Math. Soc. 298 (1986), 779-791.

[6] P. F. Leung, On the topology of a compact submanifold of a sphere with bounded second fundamental form, Manus. Math. 79 (1993), 183-185.

[7] H. W. Xu and W. Fang, Geometrical and topological rigidity of complete submanifolds, Preprint, 2001.

[8] M. Micallef and J. D. Moore, Minimal two-spheres and the topology of manifolds with positive curvature on totally isotropic two-planes, Ann. Math. 127 (1988), 199-227.

[9] H. W. Chen, Pointwise 1/4-pinched 4-manifolds, Ann. Global Anal. Geom. 9 (1991), 161-176.

[10] S. Brendle and R. Schoen, Manifolds with 1/4-pinched curvature are space forms, J. Am. Math. Soc. 22 (2009), 287-307.

[11] S. Brendle and R. Schoen, Classification of manifolds with weakly 1/4pinched curvatures, Acta Math. 200 (2008), 1-13.

[12] S. Brendle, A general convergence result for the Ricci flow in higher dimensions, Duke Math. J. 145 (2008), 585-601.

[13] H. P. Fu and H. W. Xu, Vanishing and topological sphere theorems for submanifolds in hyperbolic space, Intern. J. Math. 19 (2008), 811-822.

[14] R. Hamilton, Four-manifolds with positive isotropic curvature, Comm. Anal. Geom. 5 (1997), 1-92. 
[15] G. Huisken, Contracting convex hypersurfaces in Riemannian manifolds by their mean curvature, Invent. Math. 84 (1986), 463-480.

[16] G. Huisken, Deforming hypersurfaces of the sphere by their mean curvature, Math. Z. 195 (1987), 205-219.

[17] K. Shiohama, Sphere theorems, in Handbook of Differential Geometry, Vol. 1, eds. F.J.E. Dillen and L.C.A. Verstraelen, Elsevier Science B.V., Amsterdam, 2000.

[18] K. Shiohama and H. W. Xu, Lower bound for $\mathrm{L}^{\mathrm{n} / 2}$ curvature norm and its application, J. Geom. Anal. 7 (1997), 377-386.

[19] K. Shiohama and H. W. Xu, Rigidity and sphere theorems for submanifolds I, II, Kyushu J. Math. 48 (1994), 291-306; 54 (2000), 103-109.

[20] C. Y. Xia, A sphere theorem for submanifolds in a manifold with pinched positive curvature, Monatsh. Math. 124 (1997), 365-368.

[21] H. W. Xu, Mean value theorem for critical points and sphere theorems, Proceedings of the 4th International Congress of Chinese Mathematicians, Vol. 2, eds. L. Ji, K. Liu, L. Yang and S.-T. Yau, Higher Education Press, China, 2007, 203-217.

[22] R. Hamilton, Three-manifolds with positive Ricci curvature, J. Differ. Geom. 17 (1982), 255-306.

[23] C. Böhm and B. Wilking, Manifolds with positive curvature operators are space forms, Ann. Math. 167 (2008), 1079-1097.

[24] P. Li and S. T. Yau, On the parabolic kernel of the Schrödinger operator, Acta Math. 156 (1986), 153-201.

Center of Mathematical Sciences

ZHEJIANG UNIVERSITY

No. 38, Zhe Da RoAD

HANGZHOU

ZHEJIANG 310027

ChinA

E-mail address: xuhw@cms.zju.edu.cn; zhaoet@cms.zju.edu.cn

RECEIVED APRIL 072009 
